\title{
Quantum Stratonovich Calculus and the Quantum Wong-Zakai Theorem
}

\author{
John Gough \\ School of Computing and Informatics, \\ Nottingham Trent University, \\ Nottingham, NG1 4BU, United Kingdom
}

\begin{abstract}
We extend the Itō-to-Stratonovich analysis or quantum stochastic differential equations, introduced by Gardiner and Collett for emission (creation), absorption (annihilation) processes, to include scattering (conservation) processes. Working within the framework of quantum stochastic calculus, we define Stratonovich calculus as an algebraic modification of the Itō one and give conditions for the existence of Stratonovich time-ordered exponentials. We show that conversion formula for the coefficients has a striking resemblance to Green's function formulae from standard perturbation theory. We show that the calculus conveniently describes the Markov limit of regular open quantum dynamical systemsin much the same way as in the Wong-Zakai approximation theorems of classical stochastic analysis. We extend previous limit results to multipledimensions with a proof that makes use of diagrammatic conventions.
\end{abstract}

\section{Introduction}

Quantum stochastic calculus [1-4] was developed as a framework to construct concrete models of irreversible quantum dynamical systems. Prior to this, models tended to consider a system couple to an environment, but with only the system being accessible to physical measurement: as a result the environment observables were often relegated to a secondary status, leaving one with a master equation for the state of the system only [ $[5],[6]$.

Hudson and Parthasarathy 3 in 1984 presented a rigorous theory of integration with respect to processes on Bosonic (later Fermionic) Fock spaces generalizing the Itō-Doob theory of stochastic integration. In addition to integrals with respect to time, they also introduced integrals with respect to creation, annihilation and number (more generally, scattering) processes. Motivated by non-commutative Feynman-Kac formulae, they were able to describe unitary dynamical evolutions of a system coupled to the Fock space environment which 
reduced to an irreversible Markov dynamics for the system when averaged over the partial trace with respect to the Fock vacuum. Here the Schrödinger equation is replaced by a quantum stochastic differential equation (QSDE) driven by the creation, annihilation and scattering processes.

Independently, Gardiner and Collett [4 in 1985 gave the version of quantum stochastic integration for the Bosonic creation and annihilation that is best known amongst the physics community. Although they did not include the scattering processes, they did introduce several important physical concepts, in particular, they gave to the noise the status of a physical observable. This has been vital in subsequent analysis of quantum filtering and feedback where the environment can act as an apparatus/ communication channel [7]-9]. In their analysis, they also introduced the Stratonovich version of the theory by extending the usual mid-point definition to non-commuting processes. This is a natural physical choice for two reasons: unlike the Itō form, the Leibniz rule of differential calculus holds for Stratonovich differentials and so physical symmetries are more apparent; secondly, in classical analysis it is generally the case that if the model can be obtained as a singular limit of regular dynamical models, then it is the Stratonovich form that resembles the pre-limit equations the most. Historically, it was actually the latter reason that lead to Stratonovich initially introducing his modification of the Itō theory. In ordinary stochastic analysis, such results involving a central limit effect for stochastic processes are known as Wong-Zakai theorems. Our original motivation stems from quantum Markov limits [11 - 15] and the desire to understand the limit processes in a Stratonovich sense.

To give a concrete mathematical account, we start from the quantum Itō theory developed by Hudson and Parthasarathy, and deduce quantum Stratonovich calculus as an algebraic modification of the quantum Itō one which restores the Leibniz rule. An approach starting from Gardiner and Collett's input processes would have been more appealing from a physical point of view, however, we do not want to bypass questions of the mathematical status of the objects considered. Traditionally, the Stratonovich integral is defined through a midpoint Riemann sum approximation: this has been extended by Chebotarev [12] to quantum stochastic integrals, however, we emphasize that our formulation here is to define Stratonovich integrals as combinations of well-defined Itō integrals where possible. Rather than there been an unique version, we find that there are degrees of freedom in how we actually achieve this - we refer to this as a "gauge" freedom - and that the standard (symmetric) choice, corresponding to the midpoint rule, is just one possibility. We give the self-consistency formula $\mathbf{G}=\mathbf{G}_{0}+\mathbf{G}_{0} \mathbf{V G}$ relating the matrix of Itō coefficients $\mathbf{G}$ to the matrix of Stratonovich coefficients $\mathbf{G}_{0}$. (Here the "potential" $\mathbf{V}$ is half the noise covariance matrix plus the gauge.) Rather surprisingly, this has the same algebraic form as the one relating the free and perturbed Green's functions in scattering theory: a fact that we readily exploit. It is shown that the Itō coefficients of a unitary process are related to Hamiltonian Stratonovich coefficients $\left(\mathbf{G}_{0}^{\dagger}=-\mathbf{G}_{0}\right)$ and that this is true for any gauge so long as the self-consistency formula can be 
solved. This allows the interpretation that the Stratonovich calculus can be viewed as a perturbation of the Itō calculus, and vice versa.

A major motivation here is the results of [15] for a quantum Markov limit involving emission, absorption and scattering. We formulate the limit as a WongZakai result where the Stratonovich QSDE resembles the pre-limit Schrödinger equation (with gauge set by the imaginary part of the complex damping). Our key requirement is convergence of the "Neumann series" $\mathbf{G}=\sum_{n=0}^{n} \mathbf{G}_{0}\left(\mathbf{V G}_{0}\right)^{n}$. We generalize the result to multiple channel noise sources and make the proof more accessible by means of diagrammatic conventions which make the connections with the Dyson series expansion transparent. We also present the results in a fluxion notation, as an alternative to the differential increment language, which is closer to the formulation employed by Gardiner and Collett 4] and effectively generalizes their results to include scattering. This also reveals a new representation (lemma 3) for the Evans-Hudson flow maps.

\section{Quantum Stochastic Calculus}

\subsection{Quantum Processes}

Given a Hilbert space $\mathfrak{h}_{1}$, the Fock space over $\mathfrak{h}_{1}$ is the Hilbert space $\Gamma\left(\mathfrak{h}_{1}\right)$ spanned by symmetrized $n$-particle vectors $\varphi_{1} \hat{\otimes} \cdots \hat{\otimes} \varphi_{n}:=(n !)^{-1} \sum_{\sigma \in \mathfrak{S}_{n}} \varphi_{\sigma(1)} \otimes$ $\cdots \otimes \varphi_{\sigma(n)}$ for $n \geq 0$ arbitrary, $\varphi_{j} \in \mathfrak{h}_{1}$ and $\mathfrak{S}_{n}$ the group of permutations on $n$ labels. The special case $n=0$ requires the introduction of unit vector $\Omega$ called the Fock vacuum vector. The inner product on $\Gamma\left(\mathfrak{h}_{1}\right)$ is given by $\left\langle\varphi_{1} \hat{\otimes} \cdots \hat{\otimes} \varphi_{n} \mid \psi_{1} \hat{\otimes} \cdots \hat{\otimes} \psi_{n}\right\rangle=\delta_{n m}(n !)^{-1} \sum_{\sigma \in \mathfrak{S}_{n}}\left\langle\varphi_{1} \mid \psi_{\sigma(1)}\right\rangle \cdots\left\langle\varphi_{n} \mid \psi_{\sigma(n)}\right\rangle$.

The exponential vector with test function $\varphi \in \mathfrak{h}_{1}$ is defined to the Fock space vector

$$
\varepsilon(\varphi)=\sum_{n=0}^{\infty} \frac{1}{\sqrt{n !}} \underbrace{\varphi \otimes \cdots \otimes \varphi}_{n \text { fold }},
$$

and we have $\langle\varepsilon(\varphi) \mid \varepsilon(\psi)\rangle=\exp \langle\varphi \mid \psi\rangle$. If $S$ is a dense subset of $\mathfrak{h}_{1}$ then the vectors $\varepsilon(\varphi)$, with $\varphi \in S$, are total in $\Gamma\left(\mathfrak{h}_{1}\right)$, that is, the closure of the span of these vectors gives the whole Fock space.

We now take the one-particle space to be the Hilbert space of $\mathbb{C}^{N}$-valued square-integrable functions of positive time: this consists of measurable functions $\mathbf{f}=\left(f_{1}, \cdots, f_{n}\right)$ with $\int_{0}^{\infty} \sum_{i=1}^{n}\left|f_{i}(t)\right|^{2}<\infty$. An orthogonal projection $\Pi_{s}$ is defined on the one-particle space for each $s>0$ by taking $\Pi_{s} \mathbf{f}=$ $\left(\chi_{[0, s]} f_{1}, \cdots, \chi_{[0, s]} f_{n}\right)$ where $\chi_{[0, s]}$ is the indicator function for the interval $[0, s]$. An $N$-channel quantum noise source is modelled by operators processes $\left\{A_{t}^{\alpha \beta}: t>0\right\}$ acting on the corresponding Fock space $\mathfrak{F}$. For $\alpha, \beta \in\{0,1, \cdots, n\}$, these processes are defined on the domain of exponential vectors by

$$
\left\langle\varepsilon(\mathbf{f}) \mid\left\{A_{t}^{\alpha \beta}-\int_{0}^{t} f_{\alpha}^{*}(s) g_{\beta}(s) d s\right\} \varepsilon(\mathbf{g})\right\rangle=0
$$


where we include the index zero by setting $f_{0}=g_{0}=1$. We shall adopt the convention that lower case Latin indices (with the exception of $t$ and $s$ which we reserve for time!) range over the values $1, \cdots, N$ while lower case Greek indices range over $0,1, \cdots, N$. We also apply an Einstein summation convention for repeated indices over the appropriate range.

We also fix a Hilbert space $\mathfrak{h}$, called the initial space. Let $D$ be a domain in $\mathfrak{h}$ and take $S$ to be the space of bounded $\mathbb{C}^{N}$-valued functions. A family $X$. $=\left\{X_{t}: t \geq 0\right\}$ of operators on $\mathfrak{h} \otimes \mathfrak{F}$ is said to be an adapted quantum stochastic process based on $(D, S)$ if, for each $t \geq 0, X_{t} u \otimes \varepsilon(\mathbf{f})$ is defined for each $u \in D$ and $\mathbf{f}=\left(f_{1}, \cdots, f_{n}\right) \in S$ and is independent of the values $f_{i}(s)$ for $s>t$. If $X^{\alpha \beta}$ are adapted processes, their stochastic integral $X$. may be written as (implied summation!) $X_{t}=\int_{0}^{t} a_{\alpha}^{\dagger}(s) X_{s}^{\alpha \beta} a_{\beta}(s) d s$, with the meaning that

$$
\begin{aligned}
&\left\langle u \otimes \varepsilon(\mathbf{f}) \mid \int_{0}^{t} a_{\alpha}^{\dagger}(s) X_{s}^{\alpha \beta} a_{\beta}(s) d s v \otimes \varepsilon(\mathbf{g})\right\rangle \\
&:=\int_{0}^{t} f_{\alpha}^{*}(s)\left\langle u \otimes \varepsilon(\mathbf{f}) \mid X_{s}^{\alpha \beta} v \otimes \varepsilon(\mathbf{g})\right\rangle g_{\beta}(s) d s
\end{aligned}
$$

The stochastic integral is more often written in the form $X_{t}=\int_{0}^{t} X_{s}^{\alpha \beta} d A_{s}^{\alpha \beta}$ or equivalently $\int_{0}^{t} d A_{s}^{\alpha \beta} X_{s}^{\alpha \beta}$ with these integrals making sense as RiemannItō limits for locally square-integrable integrands. The stochastic integral will again be an adapted process. At the moment, the symbols $a_{\alpha}^{\dagger}(t), a_{\beta}(t)$ have no meaning other than notational, however $a_{0}^{\dagger}(t)$ and $a_{0}(t)$ are easily interpreted as the identity operator and $a_{i}(t)$ as a pointwise Malliavin gradient. What is crucial is that they appear in Wick order: that is, $a_{\alpha}^{\dagger}(t)$ to the left of $a_{\beta}(t)$. Let $Y_{t}=\int_{0}^{t} a_{\alpha}^{\dagger}(s) Y_{s}^{\alpha \beta} a_{\beta}(s) d s$ be a second integral, with the $Y^{\alpha \beta}$ adapted, then we have the formula [3]

$$
\begin{aligned}
X_{t} Y_{t}= & \int_{0}^{t} a_{\alpha}^{\dagger}(s) X_{s}^{\alpha \beta} Y_{s} a_{\beta}(s) d s+\int_{0}^{t} a_{\alpha}^{\dagger}(s) X_{s} Y_{s}^{\alpha \beta} a_{\beta}(s) d s \\
& +\int_{0}^{t} a_{\alpha}^{\dagger}(s) X_{s}^{\alpha \mu} P_{\mu \nu} Y_{s}^{\nu \beta} a_{\beta}(s) d s,
\end{aligned}
$$

where we introduce

$$
P^{\mu \nu}= \begin{cases}1, & \mu=\nu \neq 0 \\ 0, & \text { otherwise }\end{cases}
$$

Introducing the differential notation $d X_{t}=X_{t}^{\alpha \beta} d A_{t}^{\alpha \beta}$, etc., we may write the quantum Itō formula in the more familiar guise as

$$
d\left(X_{t} Y_{t}\right)=\left(d X_{t}\right) Y_{t}+X_{t}\left(d Y_{t}\right)+\left(d X_{t}\right)\left(d Y_{t}\right)
$$

where the Itō correction is $\left(d X_{t}\right)\left(d Y_{t}\right)=X_{t}^{\alpha i} Y_{t}^{i \beta} d A_{t}^{\alpha \beta} \equiv X_{t}^{\alpha \mu} P^{\mu \nu} Y_{t}^{\nu \beta} d A_{t}^{\alpha \beta}$. 
Let us define iterated integrals in the natural way:

$$
\begin{aligned}
&\left\langle\varepsilon(\mathbf{f}) \mid \int_{\Delta_{n}(t)} d A_{t_{n}}^{\alpha_{n} \beta_{n}} \cdots d A_{t_{1}}^{\alpha_{1} \beta_{1}} \varepsilon(\mathbf{g})\right\rangle \\
& \quad=\int_{\Delta_{n}(t)} f_{\alpha_{n}}^{*}\left(t_{n}\right) \cdots f_{\alpha_{1}}^{*}\left(t_{1}\right) g_{\beta_{n}}\left(t_{n}\right) \cdots g_{\beta_{1}}\left(t_{1}\right)\langle\varepsilon(\mathbf{f}) \mid \varepsilon(\mathbf{g})\rangle
\end{aligned}
$$

where $\Delta_{n}(t)$ is the simplex $t \geq t_{n}>\cdots \geq t_{1}>0$.

\subsection{Quantum Markov Evolutions}

Let $\left\{G_{\alpha \beta}\right\}$ be bounded operators on a fixed initial space $\mathfrak{h}$, then there exist a unique solution $U$. to the equation $U_{t}=1+\int_{0}^{t} G_{\alpha \beta} U_{s} d A_{s}^{\alpha \beta}$ which we can naturally interpret as the QSDE $d U_{t}=G_{\alpha \beta} U_{t} d A_{t}^{\alpha \beta}$, with $U_{0}=1$. In such cases, we may write $U$. as the Dyson-Itō time-ordered exponential $U_{t}=$ $\tilde{\mathbf{T}}_{I D} \exp \int_{0}^{t} G_{\alpha \beta} d A^{\alpha \beta}$.

Proposition 1: Let $U$. be the solution to the QSDE $d U_{t}=G_{\alpha \beta} U_{t} d A_{t}^{\alpha \beta}$, with $U_{0}=1$, where the $\left\{G_{\alpha \beta}\right\}$ are bounded operators on $\mathfrak{h}$. Necessary and sufficient conditions [3] for $U$. to be unitary process are that $G_{\alpha \beta}+G_{\beta \alpha}^{\dagger}+G_{i \alpha}^{\dagger} G_{i \beta}=0=$ $G_{\alpha \beta}+G_{\beta \alpha}^{\dagger}+G_{i \alpha} G_{i \beta}^{\dagger}$ with the general solution

$$
\begin{aligned}
& G_{i j}=W_{i j}-\delta_{i j} ; \quad G_{i 0}=L_{i} ; \\
& G_{0 j}=-L_{k}^{\dagger} W_{k j} ; \quad G_{00}=-\frac{1}{2} L_{k}^{\dagger} L_{k}-i H .
\end{aligned}
$$

where $W_{i j}, L_{i}$ and $H$ are bounded operators on the initial space with $H$ selfadjoint and $W_{i j}^{\dagger} W_{j k}=\delta_{i k}=W_{i j} W_{j k}^{\dagger}$.

Proposition 2: Let $U$. be the unitary process described above The corresponding flow map is given by $J_{t}(X):=U_{t}^{\dagger}(X \otimes 1) U_{t}$ for bounded $X$ on the initial space. We find that $J_{t}(X)$ satisfies the $Q S D E$

$$
d J_{t}(X)=J_{t}\left(\mathcal{L}_{\alpha \beta}(X)\right) d A_{t}^{\alpha \beta},
$$

where the Evans-Hudson maps [16] are given by $\mathcal{L}_{\alpha \beta}(X):=X G_{\alpha \beta}+G_{\beta \alpha}^{\dagger} X+$ $G_{i \alpha}^{\dagger} X G_{i \beta}$.

Following Lindblad [17, the dissipation of a linear map $\mathcal{L}$ on the algebra of bounded operators on $\mathfrak{h}$ is defined to be the bilinear mapping $\mathfrak{D} \mathcal{L}:(X, Y) \mapsto$ $\mathcal{L}(X Y)-\mathcal{L}(X) Y-X \mathcal{L}(Y)$.

Proposition 3: The Evans-Hudson maps for a unitary flow satisfy $\mathcal{L}_{\alpha \beta}(X)^{\dagger}=$ $\mathcal{L}_{\beta \alpha}\left(X^{\dagger}\right)$ and their dissipation is described by the equation

$$
\mathfrak{D} \mathcal{L}_{\alpha \beta}(X, Y)=\mathcal{L}_{\alpha \mu}(X) P^{\mu \nu} \mathcal{L}_{\nu \beta}(Y) .
$$




\subsection{Approximations}

For each $\lambda>0$, we set $a_{0}^{\#}(t, \lambda)=0$ and take $a_{i}^{\#}(t, \lambda)=A^{\#}(\varphi(i, t, \lambda))$ where $\varphi(i, t, \lambda)$ is a $\mathbb{C}^{N}$-valued square-integrable function on $[0, \infty)$ : we also take $t \rightarrow$ $\varphi(i, t, \lambda)$ to be strongly differentiable. We assume that $\langle\varphi(i, t, \lambda) \mid \varphi(j, s, \lambda)\rangle \equiv$ $C_{i j}(t-s, \lambda)$ where $C_{i j}(\tau, \lambda)=C_{j i}(-\tau)^{*}$ is integrable in $\tau$, and that we have the convergence $\lim _{\lambda \rightarrow 0} C_{i j}(\tau, \lambda)=\delta_{i j} \delta(\tau)$ in the sense of Schwartz distributions. We set $\kappa_{i j}=\lim _{\lambda \rightarrow 0} \int_{0}^{\infty} C_{i j}(\tau, \lambda) d \tau$ and note the identity $\kappa_{i j}+\kappa_{j i}^{*}=\delta_{i j}$.

The $a_{i}^{\#}(t, \lambda)$ are approximations to quantum white noises. We may introduce integrated processes $A_{t}^{\alpha \beta}(\lambda):=\int_{0}^{t} a_{\alpha}^{\dagger}(s, \lambda) a_{\beta}(s, \lambda) d s$ which serve as approximations to the fundamental processes. The approximation will be termed symmetric if $\kappa_{i j}=\frac{1}{2} \delta_{i j}$, but this is only a special case. Defining smeared exponential vectors by

$$
\varepsilon_{\lambda}(\mathbf{f})=\exp \left\{\int_{0}^{\infty} \sum_{i=1}^{N} f_{i}(t) a_{i}^{\dagger}(t) d t\right\} \Omega
$$

we see that the limit

$$
\lim _{\lambda \rightarrow 0}\left\langle\varepsilon_{\lambda}(\mathbf{f}) \mid \int_{\Delta_{n}(t)} a_{\alpha_{n}}^{*}\left(t_{n}, \lambda\right) \cdots a_{\alpha_{1}}^{*}\left(t_{1}, \lambda\right) a_{\beta_{n}}\left(t_{n}, \lambda\right) \cdots a_{\beta_{1}}\left(t_{1}, \lambda\right) \varepsilon_{\lambda}(\mathbf{g})\right\rangle
$$

coincides with (5).Therefore, whenever we consider the limit of Wick ordered expressions, it doesn't matter whether we have the symmetric approximation or not.

A term such as $\int_{\Delta_{2}(t)} a_{i}\left(t_{2}, \lambda\right) a_{j}^{\dagger}\left(t_{1}, \lambda\right)$ must, however, be put to Wick order as $\int_{\Delta_{2}(t)} a_{j}^{\dagger}\left(t_{1}, \lambda\right) a_{i}\left(t_{2}, \lambda\right)$ plus an additional term $\int_{\Delta_{2}(t)} C_{i j}\left(t_{2}-t_{2}, \lambda\right)$ which converges to $\kappa_{i j} t$. As a rule, expressions out of Wick order will have a limit that depends on the constants $\kappa_{i j}$.

\subsection{Notation and Conventions}

Let $\mathbf{X}=\left\{X_{\alpha \beta}\right\}$ be an $(N+1) \times(N+1)$ matrix of bounded operators on some Hilbert space. (Previously, we had the Itō coefficients which were operators on $\mathfrak{h} \otimes \mathfrak{F}$.) We shall adopt the matrix representation

$$
\mathbf{X}=\left(\begin{array}{c|c}
X_{00} & X_{01}, X_{02}, \cdots \\
\hline X_{10} & \mathrm{X} \\
X_{20} & \\
\vdots &
\end{array}\right)
$$

where $\mathrm{X}$ is the $N \times N$ sub-matrix consisting of the entries $\left\{X_{i j}\right\}$.

For instance, $\mathbf{P}=\left\{P^{\alpha \beta}\right\}$ will be a projection operator:

$$
\mathbf{P}=\left(\begin{array}{l|l}
0 & 0 \\
\hline 0 & 1
\end{array}\right), \mathbf{Q}=\mathbf{1}-\mathbf{P}=\left(\begin{array}{l|l}
1 & 0 \\
\hline 0 & 0
\end{array}\right)
$$


The quantum Itō correction is therefore described by the matrix $\mathbf{X}(t) \mathbf{P Y}(t) \equiv$ $\left\{X_{\alpha \mu}(t) P^{\mu \nu} Y_{\nu \beta}(t)\right\}$.

Let $\left\{G_{\alpha \beta}\right\} \equiv \mathbf{G}$ be a matrix with bounded operators on $\mathfrak{h}$ as entries, then the Dyson-Itō time-ordered exponential $U_{t}=\tilde{\mathbf{T}}_{D I}\left\{\exp \int_{0}^{t} G_{\alpha \beta} d A^{\alpha \beta}\right\}$ will be unitarity if, from proposition 1 ,

$$
\mathbf{G}+\mathbf{G}^{\dagger}+\mathbf{G}^{\dagger} \mathbf{P G}=\mathbf{0}, \quad \mathbf{G}+\mathbf{G}^{\dagger}+\mathbf{G P G}^{\dagger}=\mathbf{0} .
$$

It is relatively easy to see that the Itō coefficients then take the general form

$$
\begin{aligned}
\text { PGP } & =\mathbf{W}-\mathbf{P} \\
\mathbf{Q G P} & =-(\mathbf{P G Q})^{\dagger} \mathbf{W} \\
\mathbf{Q G Q} & =-\frac{1}{2}(\mathbf{P G Q})^{\dagger}(\mathbf{P G Q})-\mathrm{i}\left(\begin{array}{l|l}
H & 0 \\
\hline 0 & 0
\end{array}\right),
\end{aligned}
$$

where $\mathbf{W}^{\dagger} \mathbf{W}=\mathbf{P}=\mathbf{W} \mathbf{W}^{\dagger}$ (i.e., the restriction of $\mathbf{W}$ to $\mathfrak{h} \otimes \mathbb{C}^{N}$ is unitary) and $H$ is self-adjoint on $\mathfrak{h}$.

More explicitly, we may set

$$
\mathbf{W}=\left(\begin{array}{c|l}
0 & 0 \\
\hline 0 & \mathbf{W}=\left\{W_{i j}\right\}
\end{array}\right), \quad \mathbf{P G Q}=\left(\begin{array}{c|c}
0 & 0,0, \cdots \\
\hline L_{1} & \\
L_{2} & 0 \\
\vdots &
\end{array}\right)
$$

where $W_{i j}, L_{i}$ and $H$ are the operators on $\mathfrak{h}$ introduced in proposition 1.

We should remark that the restriction to a finite number $N$ of channels is not essential and that the unitary process exists under certain conditions on the boundedness of $\mathbf{G}$ as a matrix operator [21.

\section{Quantum Stratonovich Calculus}

We wish to write the quantum Itō formula in the form

$$
d\left(X_{t} Y_{t}\right)=\left(d X_{t}\right) \circ Y_{t}+X_{t} \circ\left(d Y_{t}\right)
$$

This can be achieved by formally defining

$$
\begin{array}{ll}
\left(d X_{t}\right) \circ Y_{t} & :=\left(d X_{t}\right) Y_{t}+X_{t}^{\alpha \mu} V_{t}^{\mu \nu} Y_{t}^{\nu \beta} d A_{t}^{\alpha \beta} \\
X_{t} \circ\left(d Y_{t}\right) & :=X_{t}\left(d Y_{t}\right)+X_{t}^{\alpha \mu}\left(V_{t}^{\nu \mu}\right)^{\dagger} Y_{t}^{\nu \beta} d A_{t}^{\alpha \beta},
\end{array}
$$

where the $\left\{V_{t}^{\alpha \beta}\right\}$ may in general be taken as adapted processes, however, we shall take them to be just scalar coefficients. It follows that $\left[\left(d X_{t}\right) \circ Y_{t}\right]^{\dagger}=$ $Y_{t}^{\dagger} \circ d X_{t}^{\dagger}$, and we recover the Ito formula provided we have the condition $V^{\mu \nu}+$ $\left(V^{\nu \mu}\right)^{*}=P^{\mu \nu}$. The simplest solution possible is to take $V^{\mu \nu}=\frac{1}{2} P^{\mu \nu}$ and this corresponds algebraically to the traditional Stratonovich definition of a 
differential. The general solution however takes the form $V^{\mu \nu}=\frac{1}{2} P^{\mu \nu}+\mathrm{i} Z^{\mu \nu}$, where the constants $\left\{Z^{\mu \nu}\right\}$ satisfy $\left(Z^{\mu \nu}\right)^{*}=Z^{\nu \mu}$. The appearance of these constants is similar to the ambiguity in the Tomita-Takesaki theory, and we refer to them as a gauge freedom. We shall identify the $V^{i j}$ with the constants $\kappa_{i j}$ occurring in the approximation scheme. Let us take $\mathbf{V} \equiv\left\{V^{\alpha \beta}\right\}$ to be the family of constants, then the requirement is $\mathbf{V}+\mathbf{V}^{\dagger}=\mathbf{P}$ with general solution $\mathbf{V} \equiv \frac{1}{2} \mathbf{P}+\mathrm{i} \mathbf{Z}$ where $\mathbf{Z}^{\dagger}=\mathbf{Z}$. We shall take the $\left\{Z^{\alpha \beta}\right\}$ to be scalar constants and set $Z^{00}=Z^{i 0}=Z^{0 j}=0$. This implies that $\mathbf{Z}=\mathbf{Z P}=\mathbf{P Z}$ and so $\mathbf{V}=\mathbf{V P}=\mathbf{P V}$ :

$$
\mathbf{V}=\frac{1}{2} \mathbf{P}+\mathrm{i} \mathbf{Z} \equiv\left(\begin{array}{l|l}
0 & 0 \\
\hline 0 & \mathrm{~V}
\end{array}\right)
$$

where $\mathrm{V}=\frac{1}{2}+\mathrm{iZ}$ with $\mathrm{Z}^{\dagger}=\mathrm{Z}$. Note that $\mathrm{V}$ is a normal operator with $\mathrm{V}^{\dagger}=$ $\mathrm{V}^{\dagger} \mathrm{V}=4^{-1}+\mathrm{Z}^{2}$.

It should be pointed out that we have the relation

$$
d J_{t}(X Y)=\left(d J_{t}(X)\right) \circ J_{t}(Y)+J_{t}(X) \circ\left(d J_{t}(Y)\right)
$$

which we can get either from taking differentials of the homomorphic property $J_{t}(X Y)=J_{t}(X) J_{t}(Y)$, or explicitly by noting that $\left(d J_{t}(X)\right) \circ J_{t}(Y) \equiv$ $J_{t}\left(\mathcal{L}_{\alpha \beta}(X) Y+\mathcal{L}_{\alpha \mu}(X) V^{\mu \nu} \mathcal{L}_{n \beta}(Y)\right) d A_{t}^{\alpha \beta}$, etc., and using proposition 3.

\subsection{Stratonovich-Dyson Time Ordered Exponentials}

Let us now suppose that $U$. is simultaneously the solution to the Itō QSDE $d U=(d G) U$, with $d G=G_{\alpha \beta} d A^{\alpha \beta}$ as before, and a Stratonovich QSDE (for a fixed gauge!)

$$
d U_{t}=\left(d G_{0}(t)\right) \circ U_{t}, \quad U_{0}=1
$$

with $d G_{0}=G_{\alpha \beta}^{0} d A^{\alpha \beta}$. In such cases, we may write $U$. as the Dyson-Stratonovich time-ordered exponential $U_{t}=\tilde{\mathbf{T}}_{S D}\left\{\exp \int_{0}^{t} G_{\alpha \beta}^{0} d A^{\alpha \beta}\right\}$ and shall refer to $\mathbf{G}_{0} \equiv$ $\left\{G_{\alpha \beta}^{0}\right\}$ as the matrix of Stratonovich coefficients.

Self-consistency requires that $d U=\left(d G^{0}\right) \circ U=(d G) U$ and so we should have that $d U=\left(d G^{0}\right) U+G_{\alpha \mu}^{0} V^{\mu \nu} G_{\nu \beta} U d A^{\alpha \beta}=\left(G_{\alpha \beta}^{0}+G_{\alpha \mu}^{0} V^{\mu \nu} G_{\nu \beta}\right) U d A^{\alpha \beta}$. This means that the Itō coefficients $\mathbf{G}=\left\{G_{\alpha \beta}\right\}$ are related to the Stratonovich coefficients $\mathbf{G}_{0}=\left\{G_{\alpha \beta}^{0}\right\}$ by

$$
\mathbf{G}=\mathbf{G}_{0}+\mathbf{G}_{0} \mathbf{V G}
$$

As we shall see, so long as $\mathbf{1}+\mathbf{V G P}$ is invertible, we may solve for $\mathbf{G}_{0}$ in terms of $\mathbf{G}$. Similarly, invertibility of $\mathbf{1}-\mathbf{P G}_{0} \mathbf{V}$ implies that we may write $\mathbf{G}$ in terms of $\mathbf{G}_{0}$. It might be remarked that the relation (14) also applies if we consider matrices $\mathbf{G}, \mathbf{G}_{0}$ of adapted processes. 
What is rather astonishing is that relation (14) is precisely of the form relating free and perturbed Green's functions. Let us recall briefly that if $H=H_{0}+V$ is a Hamiltonian considered as a perturbation of the free Hamiltonian $H_{0}$ then the resolvent operator $\mathcal{G}(z)=(z-H)^{-1}$ is related to the free resolvent $\mathcal{G}_{0}(z)=\left(z-H_{0}\right)^{-1}$ by the algebraic identity

$$
\mathcal{G}=\mathcal{G}_{0}+\mathcal{G}_{0} V \mathcal{G}
$$

for all $z$ outside of the spectra of $H$ and $H_{0}$. The identity may be rewritten as $\mathcal{G}=\left(1-\mathcal{G}_{0} V\right)^{-1} \mathcal{G}_{0}$ and iterated to give the formal expansion $\mathcal{G}=\mathcal{G}_{0}+\mathcal{G}_{0} V \mathcal{G}_{0}+$ $\mathcal{G}_{0} V \mathcal{G}_{0} V \mathcal{G}_{0}+\cdots$ which, when convergent, is the Neumann series. The details of the actually scattering are contained in the operator $\mathcal{T}:=V+V \mathcal{G} V$ and we have the identity $\mathcal{G}=\mathcal{G}_{0}+\mathcal{G}_{0} \mathcal{T} \mathcal{G}_{0}$.

\subsection{The T-matrix}

We now exploit the similarity between (14) and (15). We begin by introducing the operator

$$
\mathbf{T}:=\mathbf{V}+\mathbf{V G V} \equiv\left(\begin{array}{l|l}
0 & 0 \\
\hline 0 & \mathbf{T}
\end{array}\right)
$$

Assuming that $1-\mathbf{V G}_{0} \mathbf{P}$ is again invertible, we obtain the following identities

$$
\begin{aligned}
\mathbf{T} & =\frac{1}{\mathbf{1 -} \mathbf{V G}_{0} \mathbf{P}} \mathbf{V}, \\
\mathbf{G}_{0} \mathbf{T} & =\mathbf{G V}, \\
\mathbf{T} \mathbf{G}_{0} & =\mathbf{V G}, \\
\mathbf{G} & =\mathbf{G}_{0}+\mathbf{G V G}_{0}, \\
\mathbf{G} & =\mathbf{G}_{0}+\mathbf{G}_{0} \mathbf{T G}_{0} .
\end{aligned}
$$

The proof of (17) comes from writing

$$
\mathbf{T}=\mathbf{V}+\mathbf{T}\left(\mathbf{G}_{0}+\mathbf{G}_{0} \mathbf{T G}\right) \mathbf{V}=\mathbf{V}+\mathbf{V G}_{0} \mathbf{T}
$$

so that $\left(\mathbf{1}-\mathbf{V G}_{0} \mathbf{P}\right) \mathbf{T}=\mathbf{V}$. The remaining identities are just precise analogues of well-known relations for resolvent operators [22].

Combining (17) and (21) we see that $\mathbf{G}$ can be expressed in terms of $\mathbf{G}_{0}$ as

$$
\mathbf{G}=\mathbf{G}_{0}+\mathbf{G}_{0}\left(\mathbf{1}-\mathbf{P V G}_{0} \mathbf{P}\right)^{-1} \mathbf{V G}_{0} .
$$

In particular, we see that $\mathbf{G}$ is bounded. We may then invert to get

$$
\mathbf{G}_{0}=\mathbf{G}-\mathbf{G}(\mathbf{1}+\mathbf{P V G P})^{-1} \mathbf{V G} .
$$

The equations (22) and (23) reveal a remarkable duality between the Itō and Stratonovich coefficients. (Of course this just means that we may view either as a "perturbation" of the other!) 
If $\mathbf{P} \mathbf{V G}_{0} \mathbf{P}$ is a strict contraction, then we may develop a Neumann series expansion $\mathbf{G}=\mathbf{G}_{0}+\mathbf{G}_{0} \mathbf{V G}_{0}+\mathbf{G}_{0} \mathbf{V G}_{0} \mathbf{V} \mathbf{G}_{0}+\cdots=\mathbf{G}_{0} \sum_{n=0}^{\infty}\left(\mathbf{V G}_{0}\right)^{n}$.

It is convenient to introduce a related matrix

$$
\mathbf{F}=\mathbf{1}+\mathbf{T G}_{0}=\mathbf{1}+\mathbf{V G}
$$

so that $\mathbf{G}=\mathbf{G}_{0} \mathbf{F}$.

\subsection{An "Optical Theorem"}

Let us next suppose that the Stratonovich coefficients take the Hamiltonian form

$$
\mathbf{G}_{0}=-\mathrm{i} \mathbf{E}
$$

where $\mathbf{E}$ is a bounded, self-adjoint operator on $\mathfrak{h} \otimes \mathbb{C}^{N+1}$. We then have the relation $\mathbf{G}_{0}^{\dagger}=-\mathbf{G}_{0}$ and set

$$
\mathbf{P E P}=\left(\begin{array}{l|l}
0 & 0 \\
\hline 0 & \mathbf{E}
\end{array}\right)
$$

so that $\mathrm{E}$ is self-adjoint on $\mathfrak{h} \otimes \mathbb{C}^{N}$. When our invertibility condition is met, it is easy to see that matrix $T$ exists and can be written as

$$
\mathrm{T}=[1+\mathrm{iVE}]^{-1} \mathrm{~V} \equiv \frac{1}{\mathrm{~V}^{-1}+\mathrm{iE}} .
$$

(In the special case where $Z=0$, the self-adjointness of $E$ ensures that $\mathrm{V}=1+\mathrm{i} \frac{1}{2} \mathrm{E}$ is invertible by von Neumann's theorem [23. Therefore the existence of matrix $T$ is guaranteed. More generally, so long as the value 1 lies in the resolvent set of ZE, this theorem implies the existence of $\mathrm{T}$.)

The related matrix $\mathbf{F}$ then takes the form

$$
\mathbf{F}=\left(\begin{array}{l|c}
1 & 0,0, \cdots \\
\hline-\mathrm{i} T_{1 j} E_{j 0} & \\
-\mathrm{i} T_{2 j} E_{j 0} & \mathrm{~F} \\
\vdots &
\end{array}\right)
$$

with $\mathrm{F}=1-\mathrm{iTE}=\mathrm{TV}^{-1}$, so that $\mathrm{F} \equiv[1+\mathrm{iVE}]^{-1}$.

Lemma 1 ("Optical Theorem"): Re T $\geq 0$ and in particular T satisfies the identity

$$
\mathrm{T}+\mathrm{T}^{\dagger}=\mathrm{FF}^{\dagger}=\mathrm{F}^{\dagger} \mathrm{F}
$$

Proof. We have that $\mathrm{T}+\mathrm{T}^{\dagger}$ may be written as

$$
\mathrm{FV}+\mathrm{V}^{\dagger} \mathrm{F}^{\dagger}=\mathrm{F}\left[\mathrm{V}\left(1-\mathrm{iEV^{ \dagger }}\right)+(1+\mathrm{iVE}) \mathrm{V}^{\dagger}\right] \mathrm{F}^{\dagger}=\mathrm{F}\left[\mathrm{V}+\mathrm{V}^{\dagger}\right] \mathrm{F}^{\dagger}=\mathrm{FF}^{\dagger} .
$$


It is then relatively straightforward to show that

$$
\begin{aligned}
\mathrm{FF}^{\dagger} & =\frac{1}{\left(1-\mathrm{iV}^{\dagger} \mathrm{E}\right)(1+\mathrm{iEV})}=\left[1-\mathrm{EZ}-\mathrm{ZE}+\mathrm{EV}\left(\mathrm{V}^{\dagger}\right) \mathrm{E}\right]^{-1} \\
& =\frac{1}{(1+\mathrm{iEV})\left(1-\mathrm{iV}^{\dagger} \mathrm{E}\right)}=\mathrm{F}^{\dagger} \mathrm{F} .
\end{aligned}
$$

A similar calculation shows that $\operatorname{Im} \mathrm{T}=-\mathrm{TET}^{\dagger}$.

\subsection{Unitarity}

We now wish to show that the choice of Hamiltonian Stratonovich coefficients naturally leads to unitary processes.

Lemma 2: Let $\mathbf{G}_{0}^{\dagger}=-\mathbf{G}_{0}$ be bounded with $\mathbf{1}-\mathbf{V G}_{0} \mathbf{P}$ invertible, and set $G^{0}(t)=\int_{0}^{t} G_{\alpha \beta}^{0} d A^{\alpha \beta}$. Then the solution to the Stratonovich $Q S D E d U=$ $\left(d G^{0}\right) \circ U, U_{0}=1$ will be unitary.

Proof. This fact is an immediate consequence of the optical theorem (27). To establish the isometric property for the Itō coefficients, first observe that $\mathbf{G}+\mathbf{G}^{\dagger}=-\mathbf{G}_{0}\left(\mathbf{T}+\mathbf{T}^{\dagger}\right) \mathbf{G}_{0}$ while

$$
\mathbf{G P G}^{\dagger}=\mathbf{G}_{0} \mathbf{F V} \mathbf{F}^{\dagger} \mathbf{G}_{0}^{\dagger}=\mathbf{G}_{0}\left(\begin{array}{l|l}
0 & 0 \\
\hline 0 & \mathrm{FF}^{\dagger}
\end{array}\right) \mathbf{G}_{0}^{\dagger}=-\mathbf{G}_{0}\left(\mathbf{T}+\mathbf{T}^{\dagger}\right) \mathbf{G}_{0} .
$$

The isometry condition in (9) then follows from the first part of (27). The co-isometric property likewise follows from the second part.

\subsection{Changing Gauge}

Let $\mathbf{G}$ be a fixed Itō coefficient matrix related to the Stratonovich coefficient matrices $\mathbf{G}_{0}^{(a)}$ and $\mathbf{G}_{0}^{(a)}$ with gauges $\mathbf{Z}^{(a)}$ and $\mathbf{Z}^{(a)}$, respectively. The two matrices will then be related by the perturbative formula

$$
\mathbf{G}_{0}^{(a)}=\mathbf{G}_{0}^{(a)}+\mathrm{iG}_{0}^{(a)}\left(\mathbf{Z}^{(a)}-\mathbf{Z}^{a}\right) \mathbf{G}_{0}^{(a)}
$$

In particular, we can relate $\mathbf{G}_{0}^{(a)}$ for a non-zero gauge to the symmetric (gauge zero) form $\mathbf{G}_{0}^{(a)}$. As we shall see, the gauge $\mathbf{Z}$ has the physically interpretation as the imaginary part in the complex damping and in many applications this may be small [20].

\section{Wick Ordering Rule}

Let $X$. and $Y$. be quantum stochastic integrals with adapted integrands as before. In terms of our notation involving the $a_{\alpha}^{\dagger}(t), a_{\beta}(t)$, the product $X_{t} Y_{t}=$ 
$\int_{0}^{t} d s_{1} \int_{0}^{t} d s_{2} a_{\alpha}^{\dagger}\left(s_{1}\right) X_{s_{1}}^{\alpha \beta} a_{\beta}\left(s_{1}\right) a_{\mu}^{\dagger}\left(s_{2}\right) Y_{s_{2}}^{\mu \nu} a_{\nu}\left(s_{2}\right)$ is not immediately interpreted as an iterated integral since it is out of Wick order. However, the rule for achieving this is formally equivalent to the kinematic relations

$$
\left[a_{\alpha}(t), Y_{s}\right]=\left\{\begin{array}{cc}
P^{\alpha \mu} Y_{t}^{\mu \nu} a_{\nu}(t), & t<s \\
V^{\alpha \mu} Y_{t}^{\mu \nu} a_{\nu}(t), & t=s \\
0, & t>s
\end{array}\right.
$$

under the integral sign, along with its adjoint $\left[X_{t}, a_{\beta}^{\dagger}(t)\right]=\left[a_{\beta}(t), X_{s}^{\dagger}\right]^{\dagger}$. These relations can be viewed as the formal commutation relations $\left[a_{\alpha}(t), a_{\beta}^{\dagger}(s)\right]=$ $V^{\alpha \beta} \delta_{+}(t-s)+\left(V^{\beta \alpha}\right)^{*} \delta_{-}(t-s)$ at work, where the $\delta_{ \pm}$are one-sided deltafunctions: $\int \delta_{ \pm}(f) f(t)=f\left(0^{ \pm}\right)$, see e.g. [24, 25]. It is possible to interpret the $a_{\alpha}^{\#}(t)$ as quantum white noise operators, but we do not stress this point further here. At this stage, we could switch to a fluxion notation such as $\dot{X}_{t}=\frac{d X_{t}}{d t}=$ $a_{\alpha}^{\dagger}(t) X_{t}^{\alpha \beta} a_{\beta}(t)$, etc., and write the Itō formula as $\frac{d}{d t}\left(X_{t} Y_{t}\right)=\dot{X}_{t} \circ Y_{t}+X_{t} \circ \dot{Y}_{t}$ with the convention that

$$
\dot{X}_{t} \circ Y_{t} \equiv a_{\alpha}^{\dagger}(t) X_{t}^{\alpha \beta} a_{\beta}(t) Y_{t}=a_{\alpha}^{\dagger}(t) X_{t}^{\alpha \beta} Y_{t} a_{\beta}(t)+a_{\alpha}^{\dagger}(t) X_{t}^{\alpha \mu} V^{\mu \nu} Y_{t}^{\nu \beta} a_{\beta}(t) \text {. }
$$

In particular, we have the following interpretation of the results of the previous section: The equation $\dot{U}_{t}=\dot{G}_{0}(t) U_{t}$ with $\dot{G}_{0}(t)=a_{\alpha}^{\dagger}(t) G_{\alpha \beta}^{0} a_{\beta}(t)$ is out of Wick order, but can be put to Wick order as $\dot{U}_{t}=a_{\alpha}^{\dagger}(t) G_{\alpha \beta} U_{t} a_{\beta}(t)$. The relation $\left[a_{\alpha}(t), U_{t}\right]=V^{\alpha \mu} G^{\mu \nu} U_{t} a_{\nu}(t)$ implies that

$$
a_{\alpha}(t) U_{t}=\left(\delta_{\alpha \beta}+V^{\alpha \mu} G_{\mu \beta}\right) U_{t} a_{\beta}(t)=F_{\alpha \beta} U_{t} a_{\beta}(t),
$$

where $\left\{F_{\alpha \beta}\right\}$ are the components of the matrix $\mathbf{F}$ introduced in (24).

The QSDE for the unitary $U$., with $G_{\alpha \beta}^{0}=-\mathrm{i} E_{\alpha \beta}$, will then be

$$
\dot{U}_{t}=-\mathrm{i} a_{\alpha}^{\dagger}(t) E_{\alpha \beta} a_{\beta}(t) U_{t}=-\mathrm{i} a_{\alpha}^{\dagger}(t) E_{\alpha \beta} F_{\beta \nu} U_{t} a_{\nu}(t)
$$

and likewise the QSDE for the flow will be

$$
\begin{aligned}
\frac{d}{d t} J_{t}(X) & =\dot{U}_{t}^{\dagger}(X) U_{t}+U_{t}^{\dagger}(X) \dot{U}_{t} \\
& =-\mathrm{i} U_{t}^{\dagger} a_{\alpha}^{\dagger}(t)\left[X, E_{\alpha \beta}\right] a_{\beta}(t) U_{t} \\
& =-\mathrm{i} a_{\mu}^{\dagger}(t) U_{t}^{\dagger} F_{\alpha \mu}^{\dagger}\left[X, E_{\alpha \beta}\right] F_{\beta \nu} U_{t} a_{\nu}(t) .
\end{aligned}
$$

Comparison with proposition 2 suggest that $\mathcal{L}_{\mu \nu}(X)=F_{\alpha \mu}^{\dagger}\left[X, E_{\alpha \beta}\right] F_{\beta \nu}$. As this is an entirely new relation, we give an independent derivation in appendix A using only the Itō calculus.

Lemma 3: Under the conventions and notations of the previous sections, the Evans-Hudson maps take the form

$$
\mathcal{L}_{\alpha \beta}(X)=-\mathrm{i} F_{\mu \alpha}^{\dagger}\left[X, E_{\mu \nu}\right] F_{\nu \beta} .
$$




\section{Quantum Wong-Zakai Theorem}

The following is the multi-dimensional version of a result first established in [15].

Theorem: Let $a_{\alpha}^{\#}(t, \lambda), \alpha=0,1, \cdots, N$, be continuous in $t$ creation / annihilation fields for each $\lambda>0$ with $A_{t}^{\alpha \beta}(\lambda)=\int_{0}^{t} a_{\alpha}^{\dagger}(s, \lambda) a_{\beta}(s, \lambda) d s$ approximating fundamental quantum stochastic processes with internal space $\mathbb{C}^{N}$ as before, with fixed gauge matrix $\mathbf{V}=\left\{V^{\alpha \beta}\right\}$. If $\Upsilon_{t}^{(\lambda)}=E_{\alpha \beta} \otimes a_{\alpha}^{\dagger}(t, \lambda) a_{\beta}(t, \lambda)$ with $E_{\alpha \beta}^{\dagger}=E_{\beta \alpha}$ bounded operators on a fixed Hilbert space $\mathfrak{h}$ such that VE is a strict contraction, then the unitary family $U^{(\lambda)}$ and the Heisenberg $d y$ namical map $J^{(\lambda)}(X)=U{ }^{(\lambda) \dagger} X U^{(\lambda)}$, determined by the Schrödinger equation $\dot{U}_{t}^{(\lambda)}=-\mathrm{i} \Upsilon_{t}^{(\lambda)} U_{t}^{(\lambda)}, U_{0}^{(\lambda)}=1$, converge in the sense of weak matrix limits to the unitary quantum stochastic process $U$. and corresponding quantum stochastic flow $J .(X)$. The limit process $U$. is unitary adapted and satisfies the Stratonovich $Q S D E d U_{t}=-\mathrm{i} E_{\alpha \beta} U_{t} \circ d A_{t}^{\alpha \beta}, U_{0}=1$, with gauge determined by $\mathbf{V}=\left\{V^{\alpha \beta}\right\}$.

The condition $\|\mathrm{VE}\|<1$ gives convergence of the Neumann series. It also implies that $\mathbf{1}+\mathbf{V E P}$ will be invertible and therefore the Stratonovich QSDE makes sense. We will sketch the proof of this theorem in the Appendix B. Provided that the strict contractivity conditions hold, we could replace $\mathrm{E}$, and indeed $\mathrm{V}$, by suitably continuous adapted processes.

It might be remarked that there exists an analogue of this result using Fermi fields in place of Bose fields 29]. The limit QSDE changes insofar as the noises must now be Fermionic processes, however, the coefficients are exactly as before.

\subsection{Examples}

\subsubsection{Classical Wong Zakai Theorem}

As a very special example of theorem, let us take the 1-dimensional case with the pre-limit Hamiltonian $\Upsilon_{t}^{(\lambda)}$ determined by $E_{00}=H, E_{01}=E_{10}=R$ and $E_{11}=0$ with $\kappa=\frac{1}{2}$. Then the limit flow is characterized by the maps $\mathcal{L}_{11}(X)=$ $0, \mathcal{L}_{10}(X)=\mathcal{L}_{01}(X)=-i[X, R]$ and $\mathcal{L}_{00}(X)=-i[X, H]-\frac{1}{2}[[X, R], R]$. For the choices $H=\frac{1}{2}(p v(q)+v(q) p)$ and $R=\frac{1}{2}(p \sigma(q)+\sigma(q) p)$, where $v(\cdot)$ and $\sigma(\cdot)$ are Lipschitz continuous with $|v(x)|,|\sigma(x)|<C(1+|x|)$ for some constant $0<C<\infty$, and $q$ and $p$ are canonical position and momentum observables, we have that $q_{t}:=J_{t}(q)$ satisfies the essentially classical SDE $d q_{t}=$ $\left[v\left(q_{t}\right)+\sigma\left(q_{t}\right) \sigma^{\prime}\left(q_{t}\right)\right] d t+\sigma\left(q_{t}\right) d Q_{t}$. or equivalently $d q_{t}=v\left(q_{t}\right) d t+\sigma\left(q_{t}\right) \circ d Q_{t}$ where $Q_{t}=A_{t}^{10}+A_{t}^{01}$ is a copy of the Wiener process.

As a result, the theorem reduces to a classical Wong-Zakai approximation theorem which states that, since $Q_{t}^{(\lambda)}=\int_{0}^{t}\left(a^{\dagger}(s, \lambda)+a(s, \lambda)\right) d s$ is an essentially classical stochastic process that is differentiable in time $t$ and converges almost always uniformly on compact time-intervals to a Wiener process $Q_{t}$, the solution to the random ODE $\dot{X}_{t}^{(\lambda)}=v\left(X_{t}^{(\lambda)}, t\right)+\sigma\left(X_{t}^{(\lambda)}, t\right) \dot{Q}_{t}^{(\lambda)}, X_{0}^{(\lambda)}=x_{0}$ 
similarly converges to the diffusion process $X$. satisfying the Stratonovich SDE

$$
d X_{t}=v\left(X_{t}, t\right) d t+\sigma\left(X_{t}, t\right) \circ d Q_{t}, \quad X_{0}=x_{0} .
$$

\subsubsection{Quantum Diffusions}

Taking $\Upsilon_{t}^{(\lambda)}=R \otimes a^{\dagger}(t, \lambda)+R^{\dagger} \otimes a(t, \lambda)+H$ leads to the limit QSDE

$$
\begin{aligned}
d U_{t} & =-\mathrm{i}\left(R \otimes d A_{t}^{\dagger}+R^{\dagger} \otimes d A_{t}+H\right) \circ U_{t} \\
& \equiv-\mathrm{i}\left(R \otimes d A_{t}^{\dagger}+R^{\dagger} \otimes d A_{t}+H\right) U_{t}-\kappa R^{\dagger} R U_{t} d t .
\end{aligned}
$$

Note that $\operatorname{Re} \kappa=\frac{1}{2}$ so that $d J_{t}(X)=-\mathrm{i} J_{t}([X, R]) d A_{t}^{\dagger}-\mathrm{i} J_{t}\left(\left[X, R^{\dagger}\right]\right) d A_{t}+$ $J_{t}(\mathcal{L}(X)) d t$ where we set $A_{t}=A_{t}^{01}, A_{t}^{\dagger}=A_{t}^{10}$ and $\mathcal{L}(X)=\mathcal{L}_{00}(X)=$ $-\mathrm{i}\left[X, H^{\prime}\right]+\frac{1}{2}\left[R^{\dagger}, X\right] R+\frac{1}{2} R^{\dagger}[X, R]$. The new operator $H^{\prime}$ is $H+\operatorname{Im}\{\kappa\} R^{\dagger} R$ which includes an energy shift coming from the complex damping $\kappa$. The theorem then reduces to a long line of results dealing with broadband noise limits, weak coupling limits, etc., in quantum physics.

It is straightforward to extend this to describe coherent states, thermal states and squeezed states [26].

\subsubsection{Counting Processes}

Let us consider the choice $\Upsilon_{t}^{(\lambda)}=E \otimes\{a(t, \lambda)+f(t)\}^{\dagger}\{a(t, \lambda)+f(t)\}$. This is what we could consider in the vacuum as an equivalent of studying $E \otimes$ $a(t, \lambda)^{\dagger} a(t, \lambda)$ in a coherent of intensity $f$. Here we have $E_{\alpha \beta}=E f_{\alpha}^{*}(t) f_{\beta}(t)$ and the one dimensional form of the theorem yields the Ito coefficients $G_{\alpha \beta}=$ $-i E_{\alpha \beta}-\kappa E_{\alpha 1} \frac{1}{1+\mathrm{i} \kappa E_{11}} E_{1 \beta}$, which in this case reduce to $G_{\alpha \beta}(t)=\frac{-\mathrm{i} E}{1+\mathrm{i} \kappa E} f_{\alpha}^{*}(t) f_{\beta}(t)$. The Itō form of the limit QSDE is then

$$
d U_{t}=\frac{-\mathrm{i} E}{1+\mathrm{i} \kappa E} d N_{t}(f) U_{t}
$$

and we introduce $N_{t}(f)=\int_{0}^{t}\left(d A_{s}^{11}+f(s) d A_{s}^{10}+f^{*}(s) d A_{s}^{01}+|f(s)|^{2} d s\right)$ which is essentially classical and corresponds to a time-inhomogeneous Poisson process with instantaneous rate of change $\nu(t)=|f(t)|^{2}$.

\section{Appendix A (Proof of Lemma 3)}

We restrict to the $N=1$ case, as this is already somewhat involved. The multi-dimensional case does not present any more technical difficulties. Here the Evans-Hudson maps are given by

$$
\begin{aligned}
& \mathcal{L}_{11}(X)=W^{\dagger} X W-X ; \\
& \mathcal{L}_{10}(X)=W^{\dagger}[X, L] ; \mathcal{L}_{01}(X)=-\left[X, L^{\dagger}\right] W ; \\
& \mathcal{L}_{00}(X)=\frac{1}{2}\left[L^{\dagger}, X\right] L+\frac{1}{2} L^{\dagger}[X, L]-\mathrm{i}[X, H] .
\end{aligned}
$$


where the operators $W, H, L$ have the explicit forms

$$
W=\frac{1-\mathrm{i} \kappa^{*} E_{11}}{1+\mathrm{i} \kappa E_{11}}, L=-\mathrm{i} \frac{1}{1+\mathrm{i} \kappa E_{11}} E_{10}, H=E_{00}+E_{01} \operatorname{Im}\left\{\frac{\kappa}{1+\mathrm{i} \kappa E_{11}}\right\} E_{10} .
$$

The components of the matrix $F$ can also be written out in detail:

$$
F_{11}=\frac{1}{1+\mathrm{i} \kappa E_{11}}, F_{10}=-\mathrm{i} \kappa \frac{1}{1+\mathrm{i} \kappa E_{11}} E_{10}, F_{01}=0, F_{00}=1 .
$$

We now check that the relation (32) is correct by direct substitution.

For $\alpha=\beta=1$, we find after a little algebra that

$$
\begin{aligned}
\mathcal{L}_{11}(X) & =\frac{1+\mathrm{i} \kappa E_{11}}{1-\mathrm{i} \kappa^{*} E_{11}} X \frac{1-\mathrm{i} \kappa^{*} E_{11}}{1+\mathrm{i} \kappa E_{11}}-X \\
& =-\mathrm{i} \frac{1}{1-\mathrm{i} \kappa^{*} E_{11}}\left[X, E_{11}\right] \frac{1}{1+\mathrm{i} \kappa E_{11}} \\
& =-\mathrm{i} F_{\mu 1}^{\dagger}\left[X, E_{\mu \nu}\right] F_{\nu 1} .
\end{aligned}
$$

For $\alpha=1, \beta=0$, we have

$$
\mathcal{L}_{10}(X)=\frac{1+\mathrm{i} \kappa E_{11}}{1-\mathrm{i} \kappa^{*} E_{11}}\left[X,-\mathrm{i} \frac{1}{1+\mathrm{i} \kappa E_{11}} E_{10}\right]
$$

and to compute this we need the observation that

$$
\left[X, \frac{1}{1+\mathrm{i} \kappa E_{11}}\right]=-\mathrm{i} \kappa \frac{1}{1+\mathrm{i} \kappa E_{11}}\left[X, E_{11}\right] \frac{1}{1+\mathrm{i} \kappa E_{11}}
$$

to write

$$
\begin{aligned}
\mathcal{L}_{10}(X) & =i \kappa \frac{1}{1-\mathrm{i} \kappa^{*} E_{11}}\left[X, E_{11}\right] \frac{1}{1+\mathrm{i} \kappa E_{11}}-\mathrm{i} \frac{1}{1-\mathrm{i} \kappa^{*} E_{11}}\left[X, E_{10}\right] \\
& =-\mathrm{i} F_{\mu 1}^{\dagger}\left[X, E_{\mu \nu}\right] F_{\nu 0} .
\end{aligned}
$$

As we have $\mathcal{L}_{\alpha \beta}(X)^{\dagger}=\mathcal{L}_{\beta \alpha}\left(X^{\dagger}\right)$, this gives the $\mathcal{L}_{01}(X)$ result also.

The final case is the Lindbladian map $\mathcal{L}_{00}$. Substituting in gives

$$
\begin{aligned}
\mathcal{L}_{00}(X) & =-\mathrm{i}\left[X, E_{00}\right]-\mathrm{i}\left[X, E_{01} \operatorname{Im}\{T\} E_{10}\right] \\
+\frac{1}{2}\left[E_{01} \frac{1}{1-\mathrm{i} \kappa^{*} E_{11}}, X\right] & \frac{1}{1+\mathrm{i} \kappa E_{11}} E_{10}+\frac{1}{2} E_{01} \frac{1}{1-\mathrm{i} \kappa^{*} E_{11}}\left[X, \frac{1}{1+\mathrm{i} \kappa E_{11}} E_{10}\right]
\end{aligned}
$$

and again we use (36) along with the observation that

$$
\operatorname{Re} T=\frac{1}{2} \frac{1}{1-\mathrm{i} \kappa^{*} E_{11}} \frac{1}{1+\mathrm{i} \kappa E_{11}}
$$

to obtain

$$
\begin{gathered}
\mathcal{L}_{00}(X)=-\mathrm{i}\left[X, E_{00}\right]-\left[X, E_{01}\right] T E_{10}+E_{01} T^{\dagger}\left[X, E_{10}\right] \\
-\mathrm{i} E_{01}[X, \operatorname{Im} T] E_{10}-\mathrm{i} E_{01} T^{\dagger}\left[X, E_{11}\right] \operatorname{Re}\{T\} E_{10}-\mathrm{i} E_{01} \operatorname{Re}\left\{T^{\dagger}\right\}\left[X, E_{11}\right] T E_{10} .
\end{gathered}
$$


The first three terms are $-\mathrm{i} F_{\alpha 0}^{\dagger}\left[X, E_{\alpha \beta}\right] F_{\beta 0}$, for $(\alpha, \beta)=(0,0),(0,1)$ and $(1,0)$ respectively. To tidy up the last term, we note the identities

$$
[X, T]=-\mathrm{i} T\left[X, E_{11}\right] T,\left[X, T^{\dagger}\right]=+\mathrm{i} T^{\dagger}\left[X, E_{11}\right] T^{\dagger}
$$

so that

$$
\begin{aligned}
& {[X, \operatorname{Im} T]+T^{\dagger}\left[X, E_{11}\right] \operatorname{Re} T+\operatorname{Re} T\left[X, E_{11}\right] T } \\
= & -\frac{1}{2} T\left[X, E_{11}\right] T-\frac{1}{2} T^{\dagger}\left[X, E_{11}\right] T+T^{\dagger}\left[X, E_{11}\right] \operatorname{Re} T+\operatorname{Re} T\left[X, E_{11}\right] T \\
\equiv & T^{\dagger}\left[X, E_{11}\right] T
\end{aligned}
$$

and therefore the last term is $-\mathrm{i} E_{01} T^{\dagger}\left[X, E_{11}\right] T E_{10} \equiv-\mathrm{i} F_{10}^{\dagger}\left[X, E_{10}\right] F_{10}$. This gives the desired form.

\section{Appendix B (Proof of the Theorem)}

We now sketch briefly the proof of limit using diagrams. Essentially, this is a form of the van Kampen cumulant expansion [20] which can be described explicitly. The Heisenberg evolution limit is similar though more involved [15].

Step 1: Wick Ordering the Dyson Series

For finite $\lambda$, the mapping $t \mapsto U_{t}^{(\lambda)}$ is differentiable, and we may formally expand as the Dyson series $U_{t}^{(\lambda)}=\sum_{n \geq 0}(-\mathrm{i})^{n} \int_{\Delta_{n}(t)} \Upsilon_{t_{n}}^{(\lambda)} \ldots \Upsilon_{t_{1}}^{(\lambda)}$. Here $\Delta_{n}(t)$ is the simplex consisting of multi-times $\left(t_{n}, \cdots, t_{1}\right)$ with $t \geq t_{n} \geq \cdots \geq t_{1} \geq 0$.

It is convenient to put the Dyson series to Wick order using the commutation relations. The most convenient way to describe this is to expand in terms of diagrammatic series and we borrow some standard techniques from field theory. To this end, we introduce four vertices corresponding to the four components of $\Upsilon_{t}^{(\lambda)}$ :

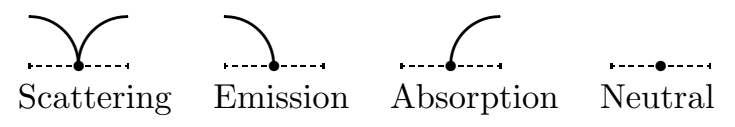

The Wick-ordered Dyson series is then given by the sum

$$
U_{t}^{(\lambda)}=\sum_{\mathfrak{D}} \hat{\mathfrak{D}}_{t}(\lambda)
$$

which we now describe. We sum over all diagrams $\mathfrak{D}$ obtained by writing $n$ vertices in a line $(n=0,1,2, \cdots)$ as below

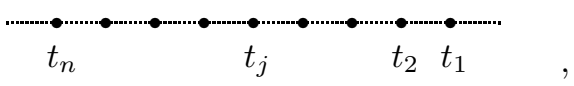


taking each of the vertices to be one of the four shown above, connecting up some of the creation/annihilation pairs and leaving the rest as external lines.

With each such diagram $\mathfrak{D}$ we associate the operator

$$
\hat{\mathfrak{D}}_{t}(\lambda)=(-\mathrm{i})^{n} E_{n} \cdots E_{1} \otimes \int_{\Delta_{n}(t)} \Pi a^{\dagger}(\lambda) \Pi C(\lambda) \Pi a(\lambda)
$$

where, depending on the type of vertex at $t_{j}$,

$$
E_{j}=\left\{\begin{array}{c}
E_{i j}, \text { scattering, } \\
E_{i 0}, \text { emission, } \\
E_{0 j}, \text { absorption, } \\
E_{00}, \text { neutral. }
\end{array}\right.
$$

and in the simplex integral $\Pi a^{\dagger}(\lambda)$ is the product over all external lines going out, $\Pi a(\lambda)$ is the product over all external lines coming in and $\Pi C(\lambda)$ is a product over all contraction pairs.

For instance the nine vertex diagram below

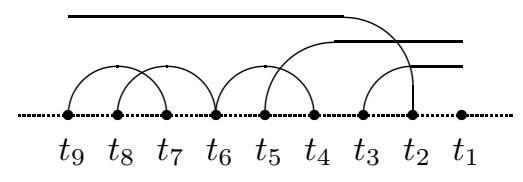

corresponds to the operator

$$
\begin{aligned}
& -\mathrm{i} E_{0 j_{9}} E_{0 j_{8}} E_{i_{7} 0} E_{i_{6} j_{6}} E_{0 j_{5}} E_{i_{4} 0} E_{0 j_{3}} E_{i_{2} 0} E_{00} \\
& \otimes \int_{\Delta_{9}(t)} a_{i_{2}}^{\dagger}\left(t_{2}, \lambda\right) a_{j_{5}}\left(t_{5}, \lambda\right) a_{j_{3}}\left(t_{3}, \lambda\right) \\
& C_{j_{9} i_{7}}\left(t_{9}-t_{7}, \lambda\right) C_{j_{8} i_{6}}\left(t_{8}-t_{6}, \lambda\right) C_{j_{6} i_{4}}\left(t_{6}-t_{4}, \lambda\right) .
\end{aligned}
$$

The diagrams we are considering are Goldstone diagrams, or time-ordered Feynman diagrams 28. The vertices however carry an operator weight $E_{\alpha \beta}$ and, even in the absence of external lines, the diagrams will not generally be scalars!

\section{Step 2: The Markov Limit of Individual Diagrams}

Let us consider the effect of the Markov limit $\lambda \rightarrow 0$ on individual diagrams. If we have a contraction

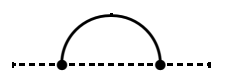

over consecutive times $t_{j+1}>t_{j}$, then we will have $t_{j+1}-t_{j} \rightarrow 0^{+}$in the limit. The effect of each such contraction is to reduce the order of the simplex by one degree and to introduce a multiplication factor $V^{i j}$. (Remember, we only get a partial contribution from the delta-function since $t_{j+1}>t_{j}$.) On the other hand, if any of the contracted time pairs are not time consecutive then the 
contribution converges weakly to zero! The reason is essentially that not just the emission and absorption times, but all the intermediate times are forced to be equal, and we get a collapse of the integral to zero.

We therefore say that a diagram is time-consecutive (TC) if each contraction appearing is between time consecutive vertices only.

\section{Step 3: The Vacuum Limit}

We now fix $u, v \in \mathfrak{h}$ and investigate the $\operatorname{limit}_{\lim _{\lambda \rightarrow 0}}\left\langle u \otimes \Omega\left|U_{t}^{(\lambda)}\right| v \otimes \Omega\right\rangle$ which we denote by the single vertex

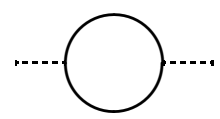

We now argue that this limit will consist of the TC diagrams having no external lines which we can express as

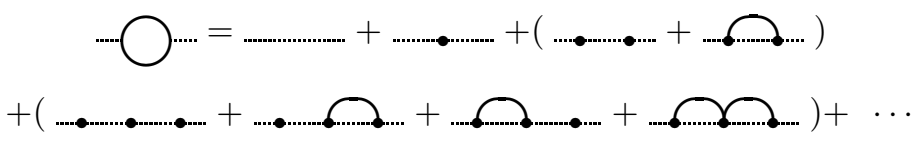

The diagrams have been grouped by vertex number, however, it also possible to group them by effective vertex number which is the actual number minus the number of contractions and which gives reduced degree of the simplex. The series can be partially re-summed as

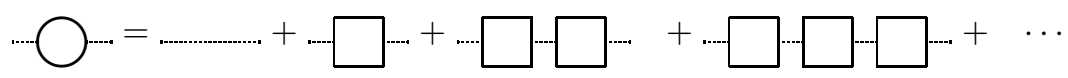

where each box is a sum over all effective one-vertex contributions:

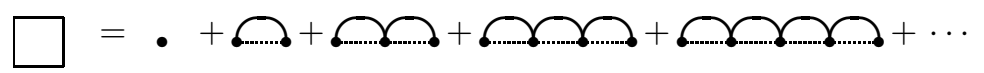

which is analogous to the expression of the self-energy in quantum field theory:as a sum over irreducible terms. (Note that series terminates at second order when there is no scattering: as this is a form of cumulant expansion, the emission/absorption problem is then Gaussian while allowing scattering means that we have cumulant moments to all orders!) Explicitly, the box at time vertex $t$ corresponds to the sum

$$
\begin{gathered}
-\mathrm{i} E_{00} d t+(-\mathrm{i})^{2} E_{0 i_{1}} E_{i_{1} 0} d t+(-\mathrm{i})^{3} E_{0 i_{4}} V^{i_{4} i_{3}} E_{i_{3} i_{2}} V^{i_{2} i_{1}} E_{i_{1} 0} d t+\cdots \\
=\left(-\mathrm{i} E_{00}-E_{0 i} V^{i j}\left(\frac{1}{1+\mathrm{iVE}}\right)_{j k} E_{k 0}\right) d t \equiv-\mathrm{i} G_{00} d t
\end{gathered}
$$

and we have summed the geometric series, convergent since $\|\mathrm{VE}\|<1$, to get the required coefficient $G_{00}$. We can write the expansion in the recursive form 


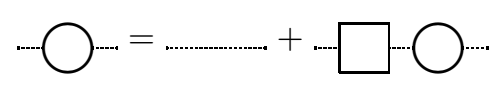

and this is interpreted as the integro-differential equation

$$
\int_{0}^{t}\left\langle u \otimes \Omega\left|d U_{t_{1}}\right| v \otimes \Omega\right\rangle=\langle u \otimes \Omega \mid v \otimes \Omega\rangle-\mathrm{i} \int_{0}^{t} d t_{2}\left\langle u \otimes \Omega\left|G_{00} \int_{0}^{t_{2}} d U_{t_{1}}\right| v \otimes \Omega\right\rangle
$$

with decaying exponential solution

$$
\langle u \otimes \Omega|U(t)| v \otimes \Omega\rangle=\left\langle u\left|e^{-\mathrm{i} t G_{00}}\right| v\right\rangle .
$$

The interpretation is intended to suggest that there is a limit object $U_{t}$ which we interpret as a unitary quantum stochastic process on a noise space with initial space $\mathfrak{h}$.

\section{Step 4: Limit of Exponential Vector Matrix Elements}

Calculating $\lim _{\lambda \rightarrow 0}\left\langle u \otimes \varepsilon_{\lambda}(\mathbf{f})|U(t, \lambda)| v \otimes \varepsilon_{\lambda}(\mathbf{g})\right\rangle$ does not require too much machinery beyond that used in the vacuum case. Indeed, we can convert the new matrix elements to vacuum ones using the relation

$$
\left\langle u \otimes \varepsilon_{\lambda}(\mathbf{f})\left|U_{t}^{(\lambda)}\right| v \otimes \varepsilon_{\lambda}(\mathbf{g})\right\rangle=\left\langle u \otimes \Omega\left|\tilde{U}_{t}^{(\lambda)}\right| v \otimes \Omega\right\rangle
$$

where $\tilde{U}_{t}^{(\lambda)}$ is the solution to the Schrödinger equation determined by the "Hamiltonian" $\tilde{\Upsilon}_{t}^{(\lambda)}$ obtained by replacing the fields $a(t, \lambda)$ and $a^{\dagger}(t, \lambda)$ with

$$
\begin{aligned}
& \tilde{a}_{i}(t, \lambda)=a_{i}(t, \lambda)+\int C_{i j}(t-s, \lambda) g_{j}(s), \\
& \tilde{a}_{i}^{\dagger}(t, \lambda)=a_{i}^{\dagger}(t, \lambda)+\left[\int C_{i j}(t-s) f_{j}(t)\right]^{*} .
\end{aligned}
$$

The new interaction $\tilde{\Upsilon}_{t}^{(\lambda)}$ isn't necessarily self-adjoint, however, that doesn't effect things. We may rearrange $\tilde{\Upsilon}_{t}^{(\lambda)}$ in terms of the original fields as $\tilde{E}_{\alpha \beta} \otimes$ $a_{\alpha}^{\dagger}(t, \lambda) a_{\beta}(t, \lambda)$ where, suppressing the $\lambda$ and $t$ dependences, the $\tilde{E}_{\alpha \beta}$ are the operators

$$
\begin{aligned}
\tilde{E}_{i j}= & E_{i j}, \\
\tilde{E}_{i 0}= & E_{i 0}+E_{i j} \int C_{j l}(t-s, \lambda) g_{l}(s), \\
\tilde{E}_{0 j}= & E_{0 j}+E_{j k}\left[\int C_{k l}(t-s) f_{l}(t)\right]^{*}, \\
\tilde{E}_{00}= & E_{00}+E_{i 0}\left[\int C_{i l}(t-s) f_{l}(t)\right]^{*}+E_{0 j} \int C_{j l}(t-s, \lambda) g_{l}(s) \\
& +E_{j k}\left[\int C_{i l}(t-s) f_{l}(t)\right]^{*} \int C_{j m}(t-s, \lambda) g_{m}(s) .
\end{aligned}
$$


Therefore we only have to repeat our previous argument, but with the original coefficients now replaced by the modified ones $\tilde{E}_{\alpha \beta}$, taking care with the $t$ and $\lambda$ dependences. This time the box at time vertex $t$ corresponds to the sum $-\mathrm{i} f_{\alpha}^{*} g_{\beta} G_{\alpha \beta} d t$ where now

$$
\begin{aligned}
G_{\alpha \beta} & =E_{\alpha \beta}-E_{\alpha i}\left(\mathrm{~V}-\mathrm{iVEV}+(-\mathrm{i})^{2} \operatorname{VEVEV}+\cdots\right)_{i j} E_{j \beta} \\
& =\left(-\mathrm{i} E_{\alpha \beta}-E_{\alpha i} V^{i j}\left(\frac{1}{1+\mathrm{iVE}}\right)_{j k} E_{k \beta}\right)
\end{aligned}
$$

and this gives the required result.

\section{Step 5: Convergence of the Series}

What we have done so far has been to expand the Dyson series, determine the asymptotic limit of each diagram term (only the TC ones survived), to replace the terms by their respective limits and to re-sum the series. To complete the proof, we need to establish that the series is absolutely and uniformly convergent. Fortunately we are able to extend proof for estimating these type of series exists for emission-absorption interactions [30] to the general case.

Let us start with the case where we have emission and absorption only in the interaction. The order must be even say, $n=2 n_{2}$, as the vacuum diagrams consist of $n_{2}$ pair contractions only. There will then be $\frac{\left(2 n_{2}\right) !}{2^{n_{2} n_{2}} !}$ such diagrams with $2 n_{2}$ vertices. A typical diagram, having $n_{2}=6$ is sketched below:

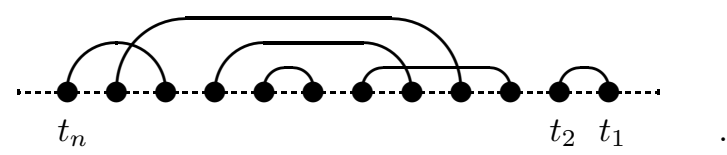

There exists a permutation $\sigma$ of the $n=2 n_{2}$ time indices which re-orders to the diagram $\mathfrak{D}_{0}(n)$ shown below

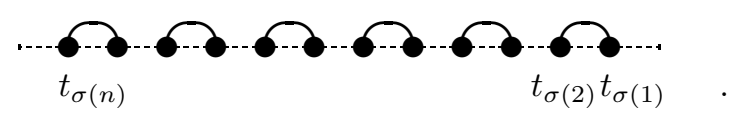

The permutation is moreover unique if it has the induced ordering of the emission times. Not all permutations arise this way, the ones that do are termed admissible. We now consider an estimate of the $n$-th term in the Dyson series. Let $E=\max \left\|E_{\alpha \beta}\right\|$, then

$$
\begin{aligned}
\left.\sum_{\mathfrak{D}} E^{n} \int_{\Delta_{n}(t)} \prod|C(\lambda)|\right|_{\mathcal{D}} & =\left.\sum_{\text {Admissible perms. }} E^{n} \int_{\Delta_{n}(t)} \prod|C(\lambda) \circ \sigma|\right|_{\mathfrak{D}_{0}(n)} \\
& =E^{n} \int_{R(t)} \prod_{k=1}^{n_{2}}\left|C\left(t_{2 k}-t_{2 k-1}, \lambda\right)\right|
\end{aligned}
$$


where $R(t)$ is the union of simplices $\left\{\left(t_{n}, \cdots, t_{1}\right): t>t_{\sigma^{-1}(n)}>\cdots>t_{\sigma^{-1}(1)}>0\right\}$ over all admissible permutations Z. $R(t)$ will be a subset of $[0, t]^{2 n_{2}}$ and if we introduce variables $t_{2 k}$ and $s_{2 k}=t_{2 k}-t_{2 k-1}$ for $k=1, \cdots, n_{2}$, it is easily seen that the above is majorized by $E^{2 n_{2}} C^{n_{2}} \times \frac{\max (t, 1)^{n_{2}}}{n_{2} !}$, where $C=\max V^{i j}$. This is the Pulè inequality [30] and clearly gives the uniform absolute estimate required to sum the series.

We now consider scattering, and constant, terms in the interaction. This time, the number of diagrams with $n$ vertices will be given by the $n$-th Bell number $B_{n}$. To see why this is so, we recall that if we have a reservoir quanta created at a vertex, then perhaps scattered, and finally reabsorbed then we can think of it as the same quantum and treat all the vertices it has been at as being linked. Each such diagram is then described by these subsets of linked vertices (we should also count the neutral vertices as these are singleton sets): in this way we have a one-to-one correspondence between the diagrams and partitions of vertices into non-empty subsets. The Bell numbers grow rapidly and have a complicated asymptotic behavior. The proliferation of diagrams is due mainly to the multiple scattering that now takes place.

Let us consider a typical diagram. We shall assume that within the diagram there are $n_{1}$ singleton vertices $[\cdots, \ldots \ldots \ldots], n_{2}$ contraction pairs $[\cdots, \ldots$. $\cdots], n_{3}$ contraction triples $[\cdots, \cdots \ldots \ldots]$, etc. That is the diagram has a total of $n=\sum_{j} j n_{j}$ vertices which are partitioned into $m=\sum_{j} n_{j}$ connected subdiagrams. For instance, we might have an initial segment of a diagram looking like the following:

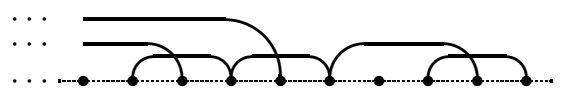

There will exist a permutation $\sigma$ of the $n$ vertices which will reorder the vertices so that we have the singletons first, then the pair contractions, then the triples, etc., so that we obtain a picture of the following type

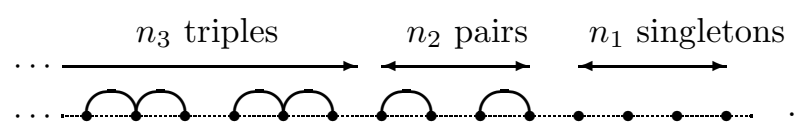

The permutation is again unique if we retain the induced ordering of the first emission times for each connected block. We now wish to find a uniform estimate for the $n$-th term in the Dyson series, we have

$$
\sum_{\mathfrak{D}} \int_{\Delta_{n}(t)} \prod|C(\lambda)| \times \text { "weights" }
$$

where the weights are the operator norms of various products of the type $E_{\alpha_{n} \beta_{n}} \cdots E_{\alpha_{1} \beta_{1}}$. Each connected diagram of $j \geq 2$ vertices will typically have 
one emission and one absorption, and $j-2$ scattering vertices. The Pulè argument of rearranging the sum over diagrams into a single integral over a region $R(t)$ of $[0, t]^{n}$ again applies and by similar reason we arrive at the upper bound, this time of the type

$$
\sum_{n_{1}, n_{2}, n_{3}, \cdots}^{\prime}\|\mathrm{VE}\|^{n_{1}+2 n_{2}+3 n_{3}+\cdots} E^{n_{1}+n_{2}+n_{3}+\cdots} \times \frac{\max (t, 1)^{n_{1}+n_{2}+n_{3}+\cdots}}{n_{1} ! n_{2} ! n_{3} ! \cdots} .
$$

Here the sum is restricted so that $\sum_{j} j n_{j}=n$. An uniform estimate for the entire series is then given by removing this restriction:

$$
\Xi(A, B)=\sum_{n_{1}, n_{2}, n_{3}, \cdots} \frac{\exp \left\{\sum_{j}(A j+B) n_{j}\right\}}{n_{1} ! n_{2} ! n_{3} ! \cdots}
$$

where $e^{A}=\|\mathrm{VE}\|$ and $e^{B}=E \max (t, 1)$. Again we use the trick to convert a sum of products into a product of sums

$$
\Xi(A, B)=\prod_{j} \sum_{n} \frac{\exp \{(A j+B) n\}}{n !}=\exp \left\{\frac{e^{A+B}}{1-e^{A}}\right\}
$$

where we need $e^{A}<1$ to sum the geometric series - this however, is precisely our condition that $\|\mathrm{VE}\|<1$.

Acknowledgment

It is a great pleasure for the author to thank the staff at MabuchiLab for their kind hospitality during his visit there when part of this paper was written. Conversations with a Hideo Mabuchi, Gerard Milburn, P. Krishnaprasad, Matthew James, Howard Wiseman, Andrew Doherty, Ramon van Handel and Luc Bouten are gratefully acknowledged.

\section{References}

[1] C. Barnett, R. F. Streater, The Itō-Clifford Integrals, I. Wilde, J. Functl. Anal., 48, 172-212 (1982)

[2] V. P. Belavkin, Theory of the control of observable quantum systems, Automatica and Remote Control 44 (2) 178-188 (1983)

[3] R. L. Hudson and K. R. Parthasarathy, Quantum Itô's formula and stochastic evolutions. Commun. Math. Phys. 93, 301-323 (1984)

[4] C. W. Gardiner and M. J. Collett, Input and output in damped quantum systems: Quantum stochastic differential equations and the master equation. Phys. Rev. A 31, 6, 3761 (1985)

[5] E. B. Davies, Quantum Theory of Open Systems (Academic Press, London, 1976) 
[6] H. Spohn, Kinetic Equations from Hamiltonian dynamics: Markovian limits, Rev. Mod. Phys. 52, 569 (1980)

[7] H.M. Wiseman, G. J. Milburn, Quantum theory of optical feedback via homodyne detection, Phys. Rev. A 49, 4110 (1994)

[8] R. van Handel, J. K. Stockton, H. Mabuchi, Feedback control of quantum state reduction, IEEE Trans. Automat. Control 50 (6), 768-780, (2005)

[9] L. Bouten, S. Edwards, V. P. Belavkin, Bellman equations for optimal feedback control of qubit states, J. Phys. B, At. Mol. Opt. Phys. 38, 151$160(2005)$

[10] J. Gough, V.P. Belavkin, O.G. Smolyanov: Hamilton-Jacobi-Bellman equations for quantum filtering and control, J. Opt. B: Quantum Semiclass. Opt. 7 S237-S244 (2005)

[11] L. Accardi, A. Frigerio, Y. G. Lu, Weak coupling limit as a quantum functional central limit theorem, Commun. Math. Phys. 131, 537-570 (1990)

[12] A. N. Chebotarev, Symmetric form of the Hudson-Parthasarathy equation. Mat. Zametki, 60, 5, 725-750 (1996)

[13] J. Gough: Asymptotic stochastic transformations for non-linear quantum dynamical systems. Reports Math. Phys. 44, No. 3, 313-338 (1999)

[14] V P Belavkin: Quantum stochastics, Dirac boundary value problem, and the ultra relativistic limit, Rep on Math. Phys. 46 (3): pp 359-381 (2000).

[15] J. Gough, Quantum flows as Markovian limit of emission, absorption and scattering interactions Commun. Math. Phys. 254, no. 2, 489-512 (2005)

[16] M. Evans, R.L. Hudson Multidimensional Quantum Diffusions Quantum Probability III, Eds. L. Accardi and W. von Waldenfels, Lecture Notes in Mathematics 1303 69-88, (1988)

[17] G. Lindblad: On the generators of completely positive semi-groups. Commun. Math. Phys 48, 119-130 (1976)

[18] B. Oksendal, Stochastic Differential Equations, Universitext, SpringerVerlag, Berlin, (1992)

[19] E. Wong, M. Zakai, On the relationship between ordinary and stochastic differential equations, Int.. J. Eng. Sci., 3, pp. 213-229 (1965)

[20] C. W. Gardiner, Quantum Noise, Springer Series in Synergetics, (1991)

[21] K. R. Parthasarathy, An Introduction to Quantum Stochastic Calculus, Monographs in Mathematics, (Birkhäuser 1992)

[22] E. N. Economou, Green's Functions in Quantum Physics, Springer Series in Solid-State Sciences, Volume 7 (1983) 
[23] M. Reed \& B. Simon, Methods of Modern Mathematical Physics, Vol 1., Theorem VIII.3, (Academic press 1972)

[24] J. Gough, Noncommumative Itô and Stratonovich noise and stochastic evolutions, Theoretical Math. Phys., 113, 276-284, (1997)

[25] W. von Waldenfels, Symmetric differentiation and Hamiltonian of a quantum stochastic process, Inf. Dim. Anal. \& Quantum Prob. 8, No. 1, 73-116, (2005)

[26] J.Gough, Quantum White Noise and the Master Equation for Gaussian Reference States, Russ. Journ. Math. Phys., 10, No.2, 142-148, (2003)

[27] W. H Louisell, Quantum Statistical Properties of Radiation, (Wiley Classics Library 1990)

[28] R. Mattuck, A Guide to Feynman Diagrams in the Many-Body Problem, (Dover Science Books 1992)

[29] J. Gough, A. Sobolev, Quantum Markovian approximations for Fermionic reservoirs, Inf. Dim. Anal. \& Quantum Prob. 8, No. 3, 453-471, (2005)

[30] J. V. Pulé, The Bloch equations, Commun. Math. Phys. 38, 241-256 (1974) 